\title{
Non-equilibrium critical dynamics of low-dimensional magnetics and multilayer structures
}

\author{
Pavel V. Prudnikov ${ }^{1 \star \star}$, Vladimir V. Prudnikov ${ }^{1}$, Alena Yu. Danilova ${ }^{1}$, Vadim O. Borzilov ${ }^{1}$, and Georgy G. Baksheev ${ }^{2}$ \\ ${ }^{1}$ Omsk State University, Pr. Mira 55A, Omsk 644077, Russia \\ ${ }^{2}$ Novosibirsk State University, Pirogova str. 1, Novosibirsk 630090, Russia
}

\begin{abstract}
The Monte Carlo simulation of the critical behavior of multilayer structures based on anisotropic Heisenberg model is performed. The influence of the uniaxial anisotropy on the critical behavior of the thin Heisenberg-like film is described. The investigation of non-equilibrium critical behavior of multilayer structure which correspond to the nanoscale superlattice $\mathrm{Co} / \mathrm{Cu}$ demonstrates that the aging effects can be observed in a wider temperature range than for bulk magnetic systems.
\end{abstract}

\section{Introduction}

Magnetic order in the multilayers is complex due to a strong influence of the shape and the magnetocrystalline anisotropies of the sample. It is well known now the fundamental role of competing interactions in the emerging features of low-dimensional systems. Among a wide number of numerical and theoretical investigations on equilibrium and dynamical properties of several model Hamiltonian of low-dimensional magnets, Heisenberg-like models are one of the most widely used to approach real magnetic materials [1].

This study includes the Monte Carlo simulation of the critical behavior of thin films and multilayers based on anisotropic Heisenberg model [2].

\section{Model and methods}

Magnetic film is constructed as a spin lattice with anisotropic Heisenberg Hamiltonian

$$
H=-J \sum_{i, j}\left[S_{i} S_{j}-\Delta(N) S_{i}^{z} S_{j}^{z}\right]-A \sum_{i}\left(S_{i}^{z}\right)^{2}-h \sum_{i} S_{i}^{x},
$$

where $\mathbf{S}_{i}$ is a three-dimensional spin in the lattice cite $i, J$ is exchange constant $J / k_{b} T=1, A$ characterizes uniaxial anisotropy in out-of-plane direction, $\mathbf{h}$ is applied magnetic field.

Anisotropy parameter $\Delta(N)$ (Figure 1) characterizes the amount of anisotropy: $\Delta=0$ corresponds to the isotropic Heisenberg case, $\Delta=1-$ the case of XY-model. Microscopic nature of anisotropy in films of $\mathrm{Fe}, \mathrm{Co}, \mathrm{Ni}$ and it's dependence from film thicknesses $N$ is determined by influence of crystalline field of substrate surface, magnetic single-ion anisotropy $[2,4]$.

\footnotetext{
^e-mail: prudnikovpv@omsu.ru
}

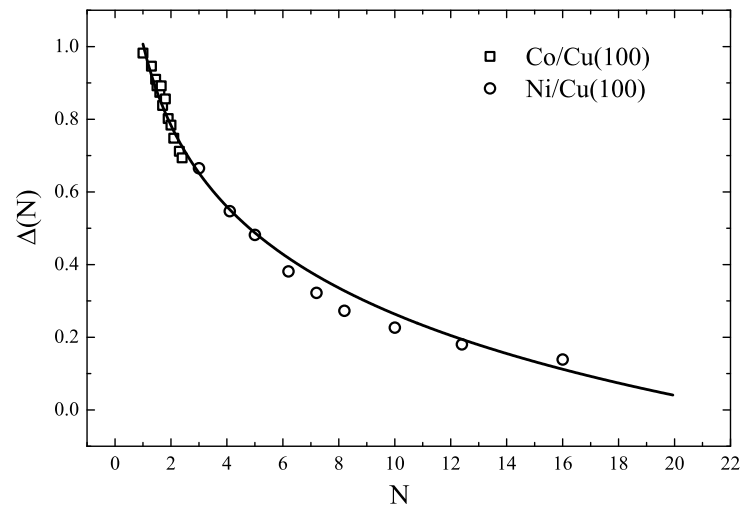

Figure 1. Anisotropy parameter $\Delta(N)$ versus the thickness of the film $N$. The dotes are corresponds to experimental data [3]

\subsection{Influence of uniaxial anisotropy on critical behavior of thin Heisenberg films}

We calculate the equilibrium characteristics of the thin Heisenberg films with the aim to to investigate the influence of uniaxial anisotropy on the critical behavior. We used values $A=0.1$ and 1.0, $\Delta=0$. For accurate determination of the critical temperatures, we consider films with different thicknesses $N=1 \mathrm{ML}, 3 \mathrm{ML}, 10 \mathrm{ML}$ and linear sizes $L=32,48,64$. Open boundary conditions were used for $z$ axis, which perpendicular to plane of films and periodic boundary conditions $\mathrm{w}$ ere used in $x y$ plane. The Metropolis algorithm was used for Monte Carlo simulations. Magnetic properties are calculated for these systems with values of $A=0.1 \mathrm{~J}, 1.0 \mathrm{~J}$, and $\Delta=0$. 

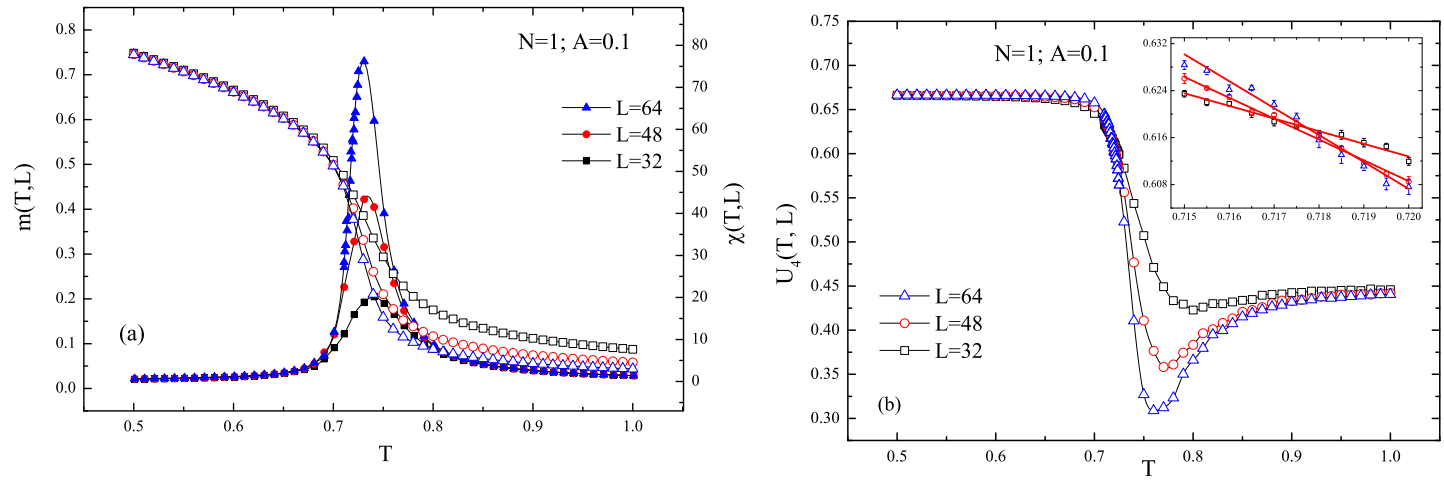

Figure 2. Temperature dependencies of the total magnetization $m(T, L)$ (open symbols), magnetic susceptibility $\chi(T, L)$ (full symbols) (a), and Binder cumulant $U_{4}(T, L)(b)$ for the film thickness $N=1$ and anisotropy $A=0.1$. The inlayer demonstrates the intersection region of the curves which determined the critical temperature $T_{c}(N)$.

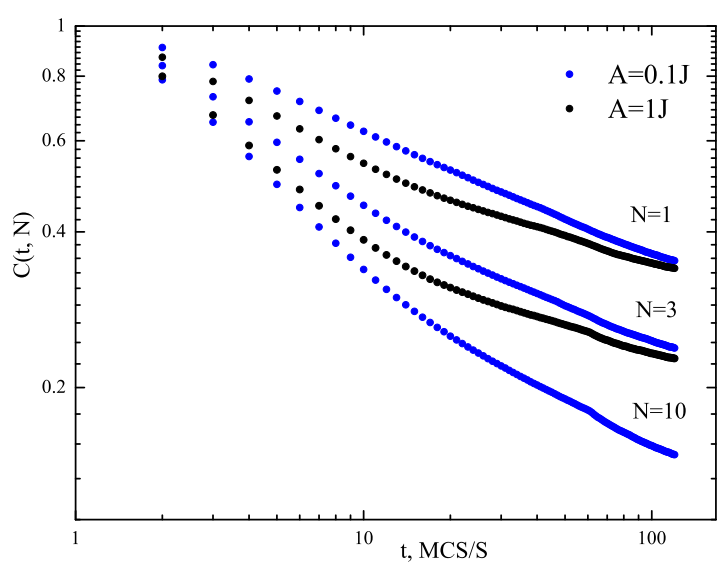

Figure 3. Non-equilibrium time dependencies of the autocorrelation function $C(t)$ for different values of $N=1,3,10$ and $A=0.1 J, 1 J$

We measured temperature dependencies of the total magnetization of film (Figure 2a)

$$
m(N, T, L)=1 / N L^{2} \sum_{i}\left(S_{i}^{x 2}+S_{i}^{y^{2}}+S_{i}^{z 2}\right)^{1 / 2} \sim L^{-\beta / v}
$$

and magnetic susceptibility (Figure 2a)

$$
\chi(N, T, L)=\frac{\left\langle m(N, T, L)^{2}\right\rangle-\langle m(N, T, L)\rangle^{2}}{T N L^{2}} \sim L^{\gamma / v},
$$

where $\langle\ldots\rangle-$ denotes the statistical averaging.

The temperature dependencies of the Binder cumulant (Figure 2b)

$$
U_{4}(N, T, L)=1 / 2\left(3-\frac{\left\langle m(N, T, L)^{4}\right\rangle}{\left\langle m(N, T, L)^{2}\right\rangle^{2}}\right) .
$$

were calculated to clarify the critical temperatures of second-order phase transition in samples. The scaling dependence of the cumulant

$$
\frac{d U_{4}}{d T}=u L^{1 / v}
$$

\begin{tabular}{|c|c|c|c|c|}
\hline \multicolumn{5}{|c|}{ Thin anisotropic films with $A=0.1 J, \Delta=0$} \\
\hline & $T_{c}, J / k_{b}$ & $v$ & $\beta$ & $\gamma$ \\
\hline$N=1$ & $0.7178(2)$ & $0.88(9)$ & $0.143(34)$ & $1.59(28)$ \\
\hline$N=3$ & $1.1842(3)$ & $0.96(10)$ & $0.159(26)$ & $1.69(26)$ \\
\hline$N=10$ & $1.4285(20)$ & $0.99(2)$ & $0.184(14)$ & $1.70(8)$ \\
\hline \multicolumn{5}{|c|}{ Thin anisotropic films with $A=1 J, \Delta=0$} \\
\hline$N=1$ & $1.005(5)$ & $1.01(6)$ & $0.134(15)$ & $1.84(15)$ \\
\hline$N=3$ & $1.483(1)$ & $0.96(7)$ & $0.103(9)$ & $1.56(15)$ \\
\hline \multicolumn{5}{|c|}{ Multilayer structure with $\Delta(N=3)=0.5677, A=0$} \\
\hline & $T_{c}, J / k_{b}$ & $v$ & $\beta$ & $\gamma$ \\
\hline & $0.784(7)$ & $0.854(31)$ & $0.626(32)$ & $0.697(42)$ \\
\hline \multicolumn{5}{|c|}{ Well-known systems } \\
\hline 2D Ising & 2.269 & 1 & 0.125 & 1.75 \\
\hline 2D XY & 0.893 & - & 0.231 & - \\
\hline 3D Ising & 4.511 & 0.630 & 0.325 & 1.241 \\
\hline $3 \mathrm{D} X Y$ & 2.202 & 0.672 & - & 1.318 \\
\hline 3D Heis. & 1.443 & 0.711 & 0.369 & 1.396 \\
\hline
\end{tabular}

Table 1. The values of the critical temperatures and exponents

makes it possible to determine the critical temperature $T_{c}(N)$ from the coordinate of the intersection points of the curves specifying the temperature dependence $U_{4}(N, T, L)$ for different $L$.

We consider finite size scaling form of film for $m(L, N)$ and $\chi(L, N)$ and use this to extract the effective critical exponents $\beta / v$ and $\gamma / v$. The scaling dependence of the cumulant allowed us to calculate values of the critical exponent $v$. The calculated values of the critical temperatures $T_{c}(N)$ and critical exponents are presented in Table 1 . We can conclude that the presence even small amount of anisotropy lead to the realization of the second order phase transition in 2D Heisenberg model. More large amount of "easy axis" anisotropy lead to the $2 \mathrm{~d}$ ising-like critical behavior with the same critical exponents, especially for $N=1$, but with different critical temperature.

The influence of the anisotropy "easy axis" on the non-equilibrium behavior can be revealed from time dependence of the autocorrelation function $C(t)=$ 

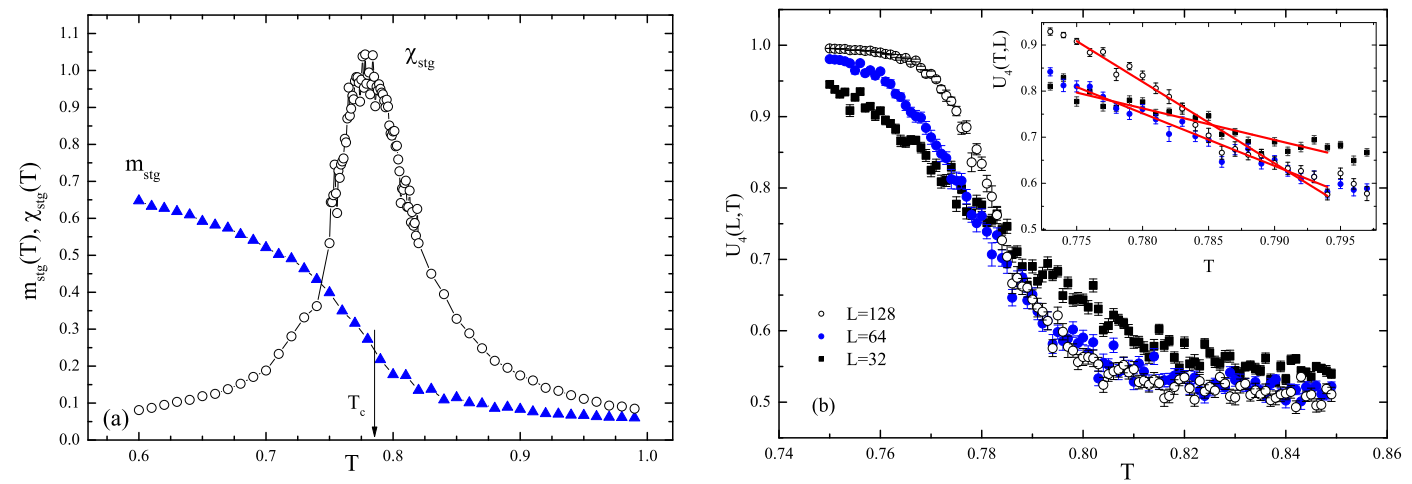

Figure 4. Temperature dependence of the of the staggered magnetization $m_{\mathrm{stg}}(T, L)$ (full symbols), magnetic susceptibility $\chi(T, L)$ (open symbols) $(a)$, and Binder cumulant $U_{4}(T, L)(b)$ for the trilayer structure
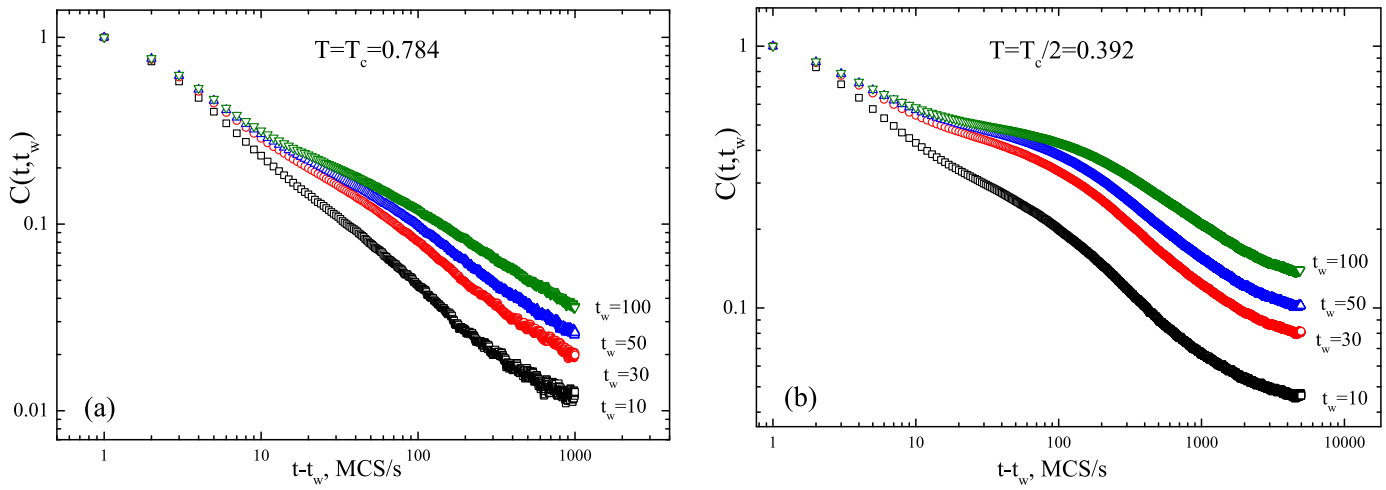

Figure 5. Autocorrelation function $C\left(t, t_{w}\right)$ versus the observation time $\left(t-t_{w}\right)$ at the evolution of the multilayer structure starting from initial state with $m_{\mathrm{stg}}^{0}=0$ for different temperatures $T=T_{c}=0.784 J_{1} / k_{b}(a)$ and $T=T_{c} / 2=0.392 J_{1} / k_{b}(b)$

$\left\langle 1 / N L^{2} \sum \boldsymbol{S}(t) \boldsymbol{S}(0)\right\rangle-\boldsymbol{m}(t) \boldsymbol{m}(0)$ (Figure 3 ). We can see that the presence of the "strong" anisotropy effects on nonequilibrium behavior only on short time interval up to 100 $\mathrm{MCS} / \mathrm{s}$. The non-equilibrium relaxation of thin anisotropic films has a strong dependence from film thickness $N$.

\subsection{Non-equilibrium critical behavior of trilayer magnetic structure}

We simulate behavior modeling of the trilayer structure consisting of two ferromagnetic films with long-range RKKI interlayer interaction separated by nonmagnetic metal film. The exchange integral $J_{1}$ determine the interaction between the neighboring spins, whereas that for the interlayer interaction is $J_{2}=-0.3 J_{1}$. The sign of $J_{2}$ is negative because the thickness of nonmagnetic spacers in multilayer structures exhibiting the giant magnetoresistance effect is tuned such that both the long-range and oscillating RKKY exchange interactions between the ferromagnetic layers effectively provide antiferromagnetism.

We measured the staggered magnetization $\boldsymbol{m}=\boldsymbol{m}_{1}$ $\boldsymbol{m}_{\mathbf{2}}$, where $\boldsymbol{m}_{1}, \boldsymbol{m}_{\mathbf{2}}$ are magnetizations of adjacent magnetic layers. The simulations were carried out for layers with sizes $L \times L \times N$ with the use of periodic and free boundary conditions for the in-plane and out-plane directions, respectively. $N$ here is the thickness of ferromagnetic layers. Magnetic properties are calculated for this trilayer with $N=3, \Delta=0.5677, A=0.0$. The equilibrium properties were simulated by the same way as in Section 2.1. The critical temperature $T_{c}=0.784(7) J_{1} / k_{b}$ for trilayer system was calculated by Binder's cumulant method (Figure 4 ).

In investigations of influence of the initial states on characteristics of non-equilibrium critical behaviour we computed the two time-dependent autocorrelation function

$$
C\left(t, t_{w}\right)=\left\langle\frac{1}{N L^{2}} \sum_{i=1}^{N L^{2}} \boldsymbol{S}_{i}(t) \boldsymbol{S}_{i}\left(t_{w}\right)\right\rangle-\boldsymbol{m}(t) \boldsymbol{m}\left(t_{w}\right),
$$

The time variable $t_{w}$ characterizes the age of a sample and is called waiting time. Autocorrelation function in nonequilibrium processes depend not only on the difference $t-t_{w}$ but also on each time variable individually at $t-t_{w}$ and $t_{w}$ much smaller than the relaxation time of the system $t_{\text {rel }}$. The non-equilibrium evolution starts when the system after initial preparation is placed to the thermostat with $T=T_{c}$. At the waiting time $t_{w}$, we have begun to measure two-time correlation function within the $1 \ll t, t_{w} \ll t_{\text {rel }}$ range. The aging of a system is manifested in slowing down relaxation processes with the time passing from the preparation of a sample. 

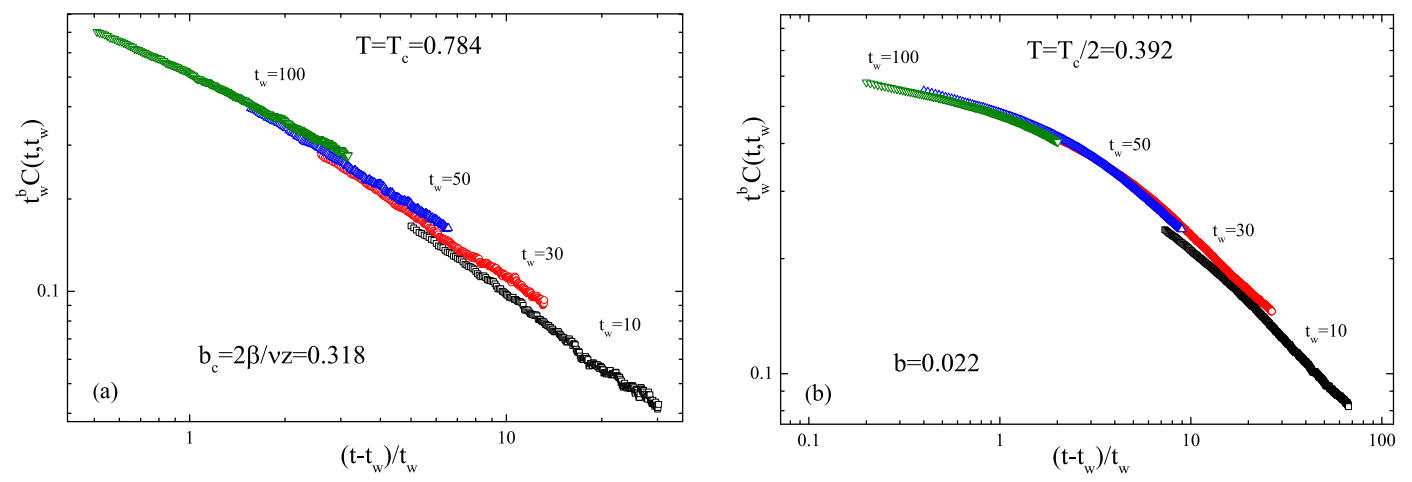

Figure 6. Scaling function $F\left(t / t_{w}\right)=t_{w}^{b} C\left(t, t_{w}\right)$ versus $\left(t-t_{w}\right) / t_{w}$ at $m_{\mathrm{stg}}^{0}=0$ for $T=T_{c}=0.784 J_{1} / k_{b}(a)$ and $T=T_{c} / 2=0.392 J_{1} / k_{b}$ (b)

For the aging regime at $t-t_{w} \sim t_{w} \gg 1$, the two-time autocorrelation function has the scaling form [5]

$$
C\left(t, t_{w}\right) \sim t_{w}^{-b} F_{C}\left(t / t_{w}\right),
$$

where the exponent $b$ at the $T_{c}$ is expressed in terms of the critical exponents as $b=2 \beta / v z$. The scaling function $F_{C}\left(t / t_{w}\right)$ is a homogeneous function of its argument $t / t_{w}$.

The features of non-equilibrium critical dynamics [5] can be considered as basis for understanding and interpretation of the experimental data [6]. The next values of modelling parameters $\Delta(N=3)=0.5677$ and $A=0$ are used for the multilayer structure, which correspond to the nanoscale superlattice $[\mathrm{Co} / \mathrm{Cu}]$ [3]. The investigation of the autocorrelation function relaxation in the multilayer superlattice has revealed the magnetic aging phenomena (Figure 5). The realization of dynamical scaling which demonstrated in Figure 6 gives the possibility to determine the critical exponent $b_{c}=2 \beta / v z=0.318$. Obtained before static critical exponents (Table 1) gives the possibility to estimate a effective system dimension $d_{e} f f=\gamma / v+2 \beta / v=2.28$. Effective exponents demonstrates dimensionality crossover from $2 \mathrm{~d} X Y$-model to 3D isotropic Heisenberg model.

\section{Conclusions}

Therefore we have studied the magnetic properties of the trilayer structure consisting of two ferromagnetic films separated by nonmagnetic metal film. The study of aging effects in multilayered structures shows that these effects brightly appear in non-equilibrium relaxation from initial states.

The nanoscale periodicity creates in these magnetic multilayer structures mesoscopic spatial magnetic correlations with slow relaxation dynamics when quenching of the system into a non-equilibrium state is realized. In comparison with bulk magnetic systems where the slow dynamics and aging phenomenon are displayed close to critical point, the magnetic superlattices, structured on the nanoscale, give possibility to increase relaxation time due to the increasing in the characteristic spin-spin correlation length. The aging can be observed in the multilayer structures in a wider temperature range $T<T c$ than for bulk magnetic systems.

\section{Acknowledgments}

This work was supported by Grant No. MD-6024.2016.2 of Russian Federation President and by Grant No. 1702-00279 of Russian Foundation for Basic Research. The simulations were supported by the Laboratory of Applied Theoretical Physics and Parallel Computation OmSU ( $2 \times$ IBM P590), the Supercomputing Center of Lomonosov Moscow State University, Moscow and Saint Petersburg Joint Supercomputer Center of the Russian Academy of Sciences.

\section{References}

[1] C.A.F. Vaz, J.A.C. Bland, G. Lauhoff, Rep. Prog. Phys. 71, 056501 (2008)

[2] P.V. Prudnikov, V.V. Prudnikov, M.A. Medvedeva, JETP Lett. 100, 446 (2014)

[3] F. Huang, M.T. Kief, G.J. Mankey, R.F. Willis, Phys. Rev. B 49, 3962 (1994)

[4] P. Prudnikov, V. Prudnikov, M. Menshikova, N. Piskunova, J. Magn. Magn. Mater. 387, 77 (2015)

[5] V. Prudnikov, P. Prudnikov, E. Pospelov, J. Stat. Mech. 043303 (2016)

[6] T. Mukherjee, M. Pleimling, Ch. Binek, Phys. Rev. B 82, 134425 (2010) 